\title{
Infecção por $C$. psittaci: uma revisão com ênfase em psitacídeos
}

\author{
C. psittaci infection: a review with emphasis in psittacines
}

\section{Laila Maftoum Proença ${ }^{\mathrm{I}}$ José Jurandir FagliariII $^{\mathrm{II}}$ Tânia de Freitas Raso ${ }^{\mathrm{II}}$}

\section{- REVISÃO BIBLIOGRÁFICA -}

\section{RESUMO}

\begin{abstract}
A clamidiose ou ornitose é uma doença infecciosa, causada pela bactéria Chlamydophila psittaci, que acomete aves e mamíferos. Trata-se de uma das principais zoonoses de origem aviária. A transmissão ocorre principalmente por inalação de secreções contaminadas. Os sinais clínicos mais comuns incluem alterações no sistema gastrointestinal, respiratório e ocular, porém é possível encontrar aves infectadas sem sinais aparentes, dificultando a identificação da doença. O diagnóstico definitivo em aves vivas pode ser difícil, devido às características da infecção pela bactéria. Há duas principais abordagens para o diagnóstico, a primeira envolve a detecção direta da bactéria e a segunda implica a detecção de anticorpos anti-Chlamydophila sp. O tratamento é longo e envolve o uso de tetraciclinas, quinolonas ou macrolídeos, durante 21-45 dias, dependendo da espécie e do fármaco de escolha. Atualmente, o Brasil não dispõe de medidas padronizadas que visam a guiar o clínico na identificação, manejo e tratamento para a doença. Tais medidas tornam-se necessárias, bem como a pesquisa de novos métodos diagnósticos e auxiliares para a doença.
\end{abstract}

Palavras-chave: Chlamydophila psittaci, zoonose aviária, clamidiose, psitacose, psitacídeos.

\section{ABSTRACT}

Chlamydiosis or ornitosis is an infectious disease which affects birds and mammals caused by the bacteria Chlamydophila psittaci. It is one of the most important avian zoonosis. The transmission occurs through inhalation of infected secretions. The most common clinical signs include problems in the gastrointestinal, respiratory and ocular tracts. However, it is possible to find infected birds with no clinical signs, which hinders the diagnosis. Definitive diagnosis in live birds can be difficult, because of the bacteria's infection characteristic. There are two main approaches to the diagnosis, the first one involves the direct detection of the bacteria, the second one involves the detection of antibodies anti-Chlamydophila sp. The treatment is long and includes the use of tetraciclines, quinolones and macrolides, during 21-45 days, depending of the specie or drug of choice. Currently, Brazil has no standardized procedures to guide clinicians in the identification, management, and treatment of the disease. Such measures become necessary, as well as research on new diagnostic methods.

Key words: Chlamydophila psittaci, avian zoonosis, chlamydiosis, psittacosis, psittacine birds.

\section{INTRODUÇÃO}

A Chlamydophila psittaci (C.psittaci), agente etiológico da clamidiose, é cosmopolita e pertence à família Chlamydiceae. Classificada como bactéria intracelular obrigatória, pode causar doença clínica em aves e mamíferos, incluindo o homem (ANDERSEN \& VANROMPAY, 2003). Devido ao contato cada vez mais próximo com pessoas, as doenças aviárias de caráter zoonótico tornam-se cada vez mais preocupantes. Doenças comuns nessas espécies, como a clamidiose, possuem prevalência subestimada, devido ao seu diagnóstico definitivo ser

'Programa de Pós-graduação em Medicina Veterinária, Universidade Estadual Paulista (UNESP), Via de Acesso Prof. Paulo Donato Castellane, s/n, 14884-900, Jaboticabal, SP, Brasil. E-mail: lailap@uol.com.br. Autor para correspondência.

"Faculdade de Medicina Veterinária e Zootecnia (FMVZ), Universidade de São Paulo (USP), São Paulo, SP, Brasil. 
difícil, em virtude da complexa fisiopatologia da infecção por Chlamydophila psittaci (RASO, 2004). O diagnóstico antemorten requer uma combinação de testes e associação com sinais clínicos e histórico e, muitas vezes, representa um desafio ao médico veterinário (RASO, 2004; NASPHV, 2010). O sucesso do tratamento e prevenção da doença está intrinsecamente relacionado ao correto diagnóstico (RASO et al., 2004). Os principais aspectos da doença e seu potencial zoonótico serão discutidos na revisão que segue.

\section{DESENVOLVIMENTO}

A clamidiose ou ornitose, causada pela bactéria Chlamydophila psittaci, é uma das principais doenças infecciosas que acomete as aves da Ordem Psittaciforme. KALETA\& TADAY (2003) compilaram dados de C. psittaci em 460 espécies aviárias e apontaram a ordem Psittaciforme com o maior percentual de espécies positivas (45\%). Trata-se de uma das principais zoonoses aviárias, com ocorrência esporádica em humanos (RASO, 2004).

A doença foi primeiramente descrita em humanos por Morange em 1893, após constatação de um agente infeccioso transmitido por papagaios. Por esse motivo, recebeu o nome de psitacose, da palavra grega psittacus, que significa papagaio (MORANGE apud VANROMPAY et al., 1995).

Devido ao contato cada vez mais próximo das aves com humanos, as doenças de caráter zoonótico desse grupo tornam-se cada vez mais preocupantes. TELFER et al. (2005) determinaram os fatores de risco em um surto de psitacose e verificaram correlação positiva entre pessoas com 50 a 64 anos de idade e contato direto com secreções contaminadas de aves silvestres. Os autores compilaram, também, dados sobre o grupo de risco para a doença, que compreende pessoas com contato próximo com aves, como proprietários de aves de companhia, trabalhadores em criação de aves comerciais e/ou em linhas de processamento de carne, indivíduos que trabalham em lojas que comercializam aves e médicos veterinários.

RASO et al. (2010) realizaram o primeiro estudo soroepidemiológico para $\boldsymbol{C}$. psittaci em humanos no Brasil. A pesquisa contemplou 364 funcionários e veterinários, entre 15 e 64 anos, de 20 zoológicos no país, com resultados demonstrando 4,7\% (17/364) de pessoas sororreagentes a C.psittaci, em sete zoológicos. Vale ressaltar que os autores encontraram valores de titulação entre 16 e 256 , demonstrando a presença de infecção recente ou em andamento. Os autores sugerem que a prevalência de
C. psittaci no Brasil é subestimada, principalmente pela ausência de testes e reagentes comerciais padronizados.

De acordo com a Associação Nacional dos Veterinários de Saúde Pública do Estado (National Association of State Health Veterinarians - NASPHV, 2010), nos Estados Unidos, 66 casos da doença foram registrados em humanos, de 2005 a 2009. Casos fatais ocorrem em até $25 \%$ dos pacientes não tratados e em menos de $1 \%$ dos pacientes tratados corretamente. A dificuldade e a demora em reconhecer e tratar a enfermidade são considerados fatores de risco em pacientes humanos (MOSCHIONI et al., 2001; PETROVAY \& BALLA, 2008; NASPHV, 2010).

A transmissão da $\boldsymbol{C}$. psittaci ao homem ocorre principalmente pela inalação do microrganismo presente em penas e fezes secas ou em secreção respiratória de aves infectadas (NASPHV, 2010). A enfermidade em humanos é descrita como uma doença de início insidioso, com sintomas brandos e inespecíficos, assemelhando-se a infecções de vias aéreas superiores (MOSCHIONI et al., 2001). Os sinais clínicos típicos incluem febre, calafrios, dor de cabeça, mialgia, mal estar, tosse não produtiva acompanhada de dificuldade respiratória, além de casos graves e multissistêmicos (MOSCHIONI et al., 2001; NASPHV, 2010). Acredita-se que a transmissão entre pessoas possa ocorrer, porém é considerada rara (OLSON \& TREUTING 1994).

No Brasil, parcos são os relatos de casos clínicos e estudos de clamidiose em aves. RASO (2004), ao compilar dados de estudos da doença em aves no Brasil, mostrou a escassez de informações no país, compendiados a estudos de PACHECO \& BIER (1931), com A. aestiva em São Paulo, e seus estudos de 1999. RASO et al. (2002) demonstraram a evidência de infecção por $\boldsymbol{C}$. psittaci em aves do gênero Amazona, utilizando 95 indivíduos cativos, aparentemente saudáveis, de três estados brasileiros. A pesquisa revelou que 16,7 a $56,1 \%$ dos papagaios aparentemente saudáveis excretavam o microrganismo e apresentaram soropositividade variando de 60 a $100 \%$.

Em 2004, foi registrado um surto de clamidiose em 58 Amazona aestiva apreendidos do tráfico em São Paulo. Os animais, entre uma a duas semanas de idade, apresentaram sinais clínicos inespecíficos, com 96,5\% de mortalidade. Exames posmortem confirmaram a presença de $\boldsymbol{C}$. psittaci (RASO et al., 2004).

A presença de $\boldsymbol{C}$. psittaci foi comprovada em ninhegos de psitacídeos em vida livre por RASO et al. (2006). O estudo com 32 amostras de A. aestiva e 45 amostras de Anodorhynchus hyacinthinus de vida livre 
no Mato Grosso do Sul demonstrou resultados positivos na detecção de $\boldsymbol{C}$. psittaci em 6,3\% (2/32) dos papagaios e em $26,7 \%$ (12/45) das araras.

Agente etiológico da clamidiose em aves e de psitacose em humanos, a Chlamydophila psittaci, é atualmente classificada como uma bactéria Gramnegativa intracelular obrigatória. Foi inicialmente classificada como vírus e, por algum tempo, como riquétsia. Recentemente, a família Chlamydiaceae foi reclassificada em dois gêneros, Chlamydia e Chlamydophila, com três e seis espécies, respectivamente. Dentre as espécies do gênero Chlamydophila, está a Chlamydophila psittaci, anteriormente classificada como Chlamydia psittaci (EVERETT et al., 1999; VANROMPAY, 2000; NASPHV, 2010).

Trata-se de um cocobacilo Gram-negativo, com 200x1.500nm, ou mais, de tamanho. Seu envoltório celular é semelhante à parede celular de outras bactérias Gram-negativas, exceto por não possuírem peptideoglicano. São intracelulares obrigatórias devido à incapacidade em obter energia mediante atividades metabólicas (BIBERSTEIN \& HIRSH, 2003).

Chlamydophila psittaci possui em sua membrana externa, uma proteína imunodominante denominada "principal proteína externa de membrana" - major outer membrane protein - MOMP - com aproximadamente $60 \%$ do peso do envoltório. Outro importante constituinte da membrana é o lipopolissacarídeo clamidial - LPS. Ambos representam importantes antígenos de superfície de membrana com uso para diagnósticos específicos (ANDERSEN \& VANROMPAY, 2003).

A família Chlamydiaceae possui um ciclo de desenvolvimento bifásico único, caracterizado por três formas morfológicas distintas: corpo elementar (CE), corpo intermediário (CI) e corpo reticular (CR). O CE representa a forma infecciosa, extracelular. É pequeno $(0,3 \mu \mathrm{m})$ e metabolicamente inativo. OCR $(0,5-1,6 \mu \mathrm{m})$ é a forma intracelular, metabolicamente ativa e não infecciosa (MOULDER, 1991; ANDERSEN \& VANROMPAY, 2003).

$\mathrm{O}$ ciclo de desenvolvimento inicia-se com a endocitose do CE pelas células eucarióticas. Os CEs permanecem no interior de inclusões intracitoplasmáticas, sofrem transformação para a forma ativa (CR) e se multiplicam por divisão binária. Nesse momento, passam por um processo de maturação para novos CE e são classificados como CI ( $0,3-1,0 \mu \mathrm{m})$. Após 24-48 horas, dependendo da espécie, novas formas infectantes (CEs) podem ser liberadas por ruptura celular e iniciam um novo ciclo em uma nova célula (MOULDER, 1991;ANDERSEN \& VANROMPAY, 2003;
LONGBOTTOM \& COULTER, 2003). A infecção persistente pode ocorrer com a permanência dos CEs no citoplasma do hospedeiro (ANDERSEN \& VANROMPAY, 2003).

Os CEs possuem fraca resistência a desinfetantes comuns, como o etanol a $70 \%$ e compostos de amônia quartenária, calor e luz solar; contudo, podem permanecer viáveis por longo período em excreções secas de animais, ou por vários dias em água à temperatura ambiente (ANDERSEN \& VANROMPAY, 2003).

A C. psittaci possui oito sorovares ou sorotipos, designados como A, B, C, D, E, F, M56 e WC. Destes, seis são conhecidos por infectarem aves (A - F). Acredita-se que cada sorovar aviário está associado a um grupo diferente. Dentre eles, o sorovar A é endêmico entre os psitacídeos, podendo causar infecções esporádicas em pessoas. O sorovar D é extremamente virulento; isolado de perus, representa grande risco aos veterinários e trabalhadores em criações aviárias (ANDERSEN \& VANROMPAY, 2003; LONGBOTTOM \& COULTER, 2003).

Em aves, a transmissão ocorre principalmente por via aerógena, mediante a inalação de excretas secas e secreções nasal e ocular de aves infectadas. Outras vias de transmissão incluem ingestão de fezes contaminadas e por meio dos pais no momento da alimentação dos filhotes no ninho (PAGE, 1959; ANDERSEN \& VANROMPAY, 2003; RASO, 2004). Há evidências de transmissão vertical da bactéria pelo ovo e, menos frequentemente, por picada de insetos (VANROMPAY et al., 1995; SHEWEN, 1980). Várias espécies de aves de vida livre são fonte potencial de contaminação e seu contato com aves domésticas deve ser evitado (PAGE \& ERICKSON, 1969; RASO, 2004). Calopsitas são consideradas portadores frequentes de C.psittaci e podem excretar a bactéria por mais de um ano após a infecção ativa (GERLACH, 1994).

O período de incubação pode variar de dias a semanas, dependendo da espécie, da virulência do agente, da idade e da condição da ave. A doença clínica é induzida por fatores estressantes, associados ao manejo impróprio, como má nutrição, excesso populacional, transporte inadequado e remoção do habitat natural (FUDGE, 1996; RASO, 1999; NASPHV, 2010). Em estudo experimental com calopsitas, GUZMAN et al. (2010) detectaram o antígeno em animais infectados com a bactéria apenas no $16^{\circ}$ dia após sua inoculação, período no qual surgiram os sinais clínicos. PAGE (1959), em estudo com perus infectados experimentalmente por meio de aerosol contaminado com C. psittaci, detectou a bactéria no sistema respiratório após 4 horas; no sangue, rins, fígado e 
baço após 48 horas; e nas fezes depois de 72 horas, com sinais clínicos aparentes apenas 5 a 10 dias após a infecção.

Os sinais clínicos podem se apresentar de forma aguda, subaguda, crônica ou inaparente, dependendo do estado imunológico da ave, da espécie hospedeira, da patogenicidade do microrganismo, do grau de exposição à bactéria, da porta de entrada e da presença de outras doenças concomitantes. As formas subaguda ou crônica são típicas de espécies com baixa suscetibilidade ou infectadas com uma cepa de virulência moderada (GERLACH, 1994; ANDERSEN \& VANROMPAY, 2003).

Os sinais da doença são inespecíficos e incluem depressão, plumagem eriçada, tremores, letargia, anorexia, desidratação, blefarite, ceratoconjuntivite, sinais respiratórios, digestórios, urinários, neurológicos e óbito, entre outros. Emaciação, desidratação, urato verde-amarelado (típico de envolvimento hepático) também podem ser notados. Conjuntivite, muitas vezes recorrente, pode ser, em alguns casos, o único sinal clínico aparente (GERLACH, 1994; NASPHV, 2010).

A forma inaparente é caracterizada pela ausência de sinais clínicos evidentes, representando muitas vezes um desafio diagnóstico. É comum em aves adultas expostas a cepas de média e baixa virulência. Nessa condição, as aves permanecem como portadoras, podendo eliminar o agente de forma intermitente (GERLACH, 1994).

A taxa de morbidade varia de acordo com a virulência da cepa envolvida, de 50 a $80 \%$ e de 5 a $20 \%$, em cepas muito e pouco virulentas, respectivamente. O mesmo ocorre com a taxa de mortalidade, que pode variar de 10 a $30 \%$, quando a cepa é muito virulenta, e de 1 a $4 \%$ no caso de cepa menos virulenta (ANDERSEN \& VANROMPAY, 2003).

RASO et al. (2002) descreveram os sinais clínicos de clamidiose em 95 filhotes de A. aestiva durante um surto da doença em São Paulo. Os animais apresentavam letargia, dispnéia, penas eriçadas, anorexia, diarréia verde-amarelada, poliúria, desidratação, emagrecimento e conjuntivite, com uma taxa de mortalidade de $96,5 \%$. Vale ressaltar que os papagaios eram provenientes do tráfico e, com isso, submetidos a manejo inapropriado.

Os achados de necropsia são também inespecíficos e normalmente limitados ao fígado, baço e sacos aéreos (LONGBOTTOM \& COULTER, 2003). RASO et al. (2004) relataram os principais achados de necropsia em dez A. aestiva positivos para $\boldsymbol{C}$. psittaci, em sendo hepatomegalia (10/10), hepatomegalia com áreas brancas e difusas no parênquima (5/10), baço pálido e pequeno (8/10) e congestão esplênica (2/10).

O diagnóstico de clamidiose em aves é dificultado pela ausência de sinais clínicos patognomônicos, os quais, associados aos exames complementares (radiológico, hematológico e bioquímico), são apenas sugestivos da doença (FUDGE, 1997; RASO, 2004). Porém um diagnóstico rápido e definitivo é necessário, devido ao potencial zoonótico da infecção (LONGBOTTOM \& COULTER, 2003). O aumento na contagem de leucócitos, alterações nas atividades de enzimas hepáticas, imagens radiográficas mostrando aumento de fígado e baço, bem como alteração em sacos aéreos são indicativos da infecção por C. psittaci (LONGBOTTOM \& COULTER, 2003).

$\mathrm{O}$ isolamento da bactéria é o método recomendado para o diagnóstico de microrganismos da Família Chlamydiaceae. Em se tratando de uma bactéria intracelular obrigatória, necessita de cultura celular. Técnicas que utilizam cultura em ovos embrionados ou linhagens celulares são necessárias para avaliar a viabilidade da bactéria; ademais, facilitam sua posterior caracterização por meio de técnicas moleculares e bioquímicas. Porém, os cuidados referentes à coleta da amostra, a fim de evitar contaminações, e a necessidade de transporte adequado, somados à necessidade de laboratórios com biossegurança nível três para a manipulação da $\boldsymbol{C}$. psittaci, dificulta a realização do procedimento na rotina clínica (LEY et al., 1993; FUDGE, 1997; SACHSE et al., 2009).

Devido às dificuldades inerentes à cultura $\mathrm{e}$ ao isolamento do microrganismo, outras técnicas de diagnóstico foram desenvolvidas. Há duas principais abordagens para o diagnóstico de uma infecção por Chlamydophila spp. A primeira envolve a detecção direta da bactéria e a segunda implica a detecção de anticorpos anti-Chlamydophila sp. (SACHSE et al. 2009; NASPHV, 2010).

Os testes de detecção direta do agente são testes rápidos e não necessitam de organismos viáveis. Entretanto, resultados falso-positivos podem ocorrer por reações cruzadas com outros agentes. Falsonegativos são possíveis quando a quantidade de antígenos é insuficiente ou no caso de excreção intermitente da bactéria. Os testes rotineiramente utilizados são ensaio imunoenzimático (ELISA) e teste de anticorpos fluorescentes (SACHSE et al. 2009; NASPHV, 2010).

O ELISA foi primeiramente desenvolvido para a detecção de Chlamydia trachomatis em humanos. No entanto, como se baseia no antígeno LPS da membrana clamidial, em teoria, é também aplicável à 
infecção em animais. Os testes de fluorescência são baseados nos antígenos MOMP e LPS da membrana da bactéria e têm as mesmas vantagens e desvantagens citadas anteriormente (SACHSE et al. 2009; NASPHV, 2010).

O diagnóstico por detecção do DNA, através da PCR com sequências específicas para detecção do agente etiológico, é o único método comercial disponível para diagnóstico de $\boldsymbol{C}$. psittaci no país, apresentando alta sensibilidade e especificidade. O material a ser colhido dependerá da técnica utilizada pelo laboratório de escolha. Amostras de fezes, swabes de cloaca ou de orofaringe são as mais utilizadas. Porém, a excreção intermitente do microrganismo pode prejudicar o emprego dos métodos de detecção da bactéria, uma vez que a ave pode não estar eliminando o agente no momento da colheita, favorecendo a ocorrência de falso-negativos (RASO, 2004; GODOY, 2007). Para tanto, sugere-se a colheita de material durante dois ou três dias consecutivos, o qual deve ser mantido em meio de transporte adequado, fornecido pelo laboratório (RASO,2004).

O diagnóstico sorológico é um procedimento auxiliar aos testes de detecção de Chlamydophila. Os anticorpos anti-C. psittaci são regularmente produzidos pelo hospedeiro infectado, embora tenham pouco ou nenhum fator protetor, já que CEs podem ser liberados mesmo na presença de elevado título de anticorpos. Um resultado positivo é evidência de que a ave foi infectada com a bactéria em um dado momento, mas não indica, necessariamente, que a ave tenha uma infecção ativa. Resultados falsonegativos podem ocorrer em infecções agudas ou iniciais, em que ainda não houve tempo para soroconversão. Sorologia negativa pode, ainda, ser verificada em aves jovens ou com imunossupressão. Tratamento com antimicrobianos pode diminuir a resposta imune. Vale ressaltar que o título de $\mathrm{IgG}$ pode permanecer alto mesmo após tratamento bem sucedido (RASO, 2004; SACHSE et al., 2009; NASPHV, 2010).

A sorologia é mais confiável quando associada ao histórico, sinais clínicos e exames complementares. Porém, é considerada diagnóstica quando a titulação encontra-se aumentada quatro vezes em amostras pareadas (com intervalo de duas a quatro semanas entre coletas) ou quando associada à presença do antígeno, mesmo em animais assintomáticos (RASO, 2004; NASPHV, 2010).

Os principais testes sorológicos para clamidiose incluem o teste de aglutinação dos corpos elementares, teste da imunofluorescência indireta, ELISA e teste de fixação do complemento direto e modificado. Este último é o teste sorológico padrão para Chlamydophila, sendo o modificado o mais sensível; porém, falso-negativos são descritos em algumas espécies de aves (ANDERSEN \& VANROMPAY, 2003; RASO, 2004; SACHSE et al., 2009; NASPHV, 2010). No Brasil, não há disponibilidade de testes sorológicos no comércio.

O tratamento de escolha para clamidiose consiste do uso de tetraciclinas, sendo a doxiciclina o fármaco de eleição para o tratamento. Trata-se de um antibiótico bacteriostático e presume-se que atue apenas durante a fase de replicação da bactéria. Para tanto, protocolos longos (30-45 dias) de tratamento são recomendados, na dose de $25-50 \mathrm{mg} \mathrm{kg}^{-1}$, para a maioria das espécies, via oral a cada 24 horas. Tratamentos alternativos têm sido descritos com o uso de macrolídeos e quinolonas, dentre eles a azitromicina e enrofloxacina. SACHSE et al. (2010) demonstraram a eficácia, in vitro, do tratamento em calopsitas, com azitromicina a cada 48 horas, durante 21 dias, na dose de $40 \mathrm{mg} \mathrm{kg}^{-1}$.

Em alguns países, como os Estados Unidos da América, a clamidiose aviária é uma doença de notificação obrigatória aos órgãos de saúde pública. Recomendações de prevenção, controle e tratamento para a doença são anualmente disponibilizados na forma de um compêndio, os quais norteiam o médico veterinário nas ações a serem tomadas frente a cada situação em particular. O compêndio disponibiliza ainda um roteiro para a definição de casos clínicos, levando em consideração as dificuldades diagnósticas inerentes à complexa fisiopatologia da infecção pela bactéria (NASPHV, 2010).

\section{CONCLUSÃO}

A clamidiose é uma das principais zoonoses de origem aviária e merece destaque dentre as enfermidades infecciosas que acometem aves silvestres. O diagnóstico da doença pode ser difícil, principalmente na ausência de sinais clínicos. $\mathrm{O}$ uso combinado de mais de uma técnica diagnóstica tornase necessário, principalmente no exame de um único indivíduo. A prevalência de $\boldsymbol{C}$.psittaci nas aves de companhia no país é subestimada e a publicação de casos clínicos da doença deve ser incentivada. Atualmente, o Brasil não dispõe de medidas padronizadas que visam a guiar o clínico na identificação, manejo e tratamento para a doença. Tais medidas tornam-se necessárias, bem como a pesquisa de novos métodos diagnósticos e auxiliares. 


\section{AGRADECIMENTOS}

À Universidade Estadual Júlio de Mesquita Filho UNESP , Campus Jaboticabal e ao Conselho Nacional de Desenvolvimento Científico e Tecnológico $(\mathrm{CNPq})$ e à Fundação de Amparo à Pesquisa do Estado de São Paulo (FAPESP), pelo incentivo e apoio à pesquisa.

\section{REFERÊNCIAS}

ANDERSEN, A.A.; VANROMPAY, D. Avian Chlamydiosis (psittacosis, ornithosis). In: SAYF, Y.M. Disease of poultry. 11.ed. Ames: Iowa State University, 2003. p.863-879.

EVERETT, K.D. et al. Emended description of the order Chlamydiales, proposal of Parachlamydiaceae fam. nov. and Simkaniaceae fam. nov., each containing one monotypic genus, revised taxonomy of the family Chlamydiaceae, including a new genus and five new species, and standards for the identification of organisms. International Journal of Systematic Bacteriology, v.49, p.415-440, 1999 . Disponível em: <http://ijs.sgmjournals.org/cgi/content/abstract/ 49/2/415>. Acesso em: 24 mar. 2011. doi: 10.1099/0020771349-2-415.

FUDGE, A.M. Avian Chlamydiosis. In: ROSSKOPF, W.J. Diseases of cage and aviary birds. Baltimore: Willians \& Willians, 1997. p.572-585.

GERLACH, H. Chlamydia. In: RICHIE, B.W.et al. Avian medicine: principles and application. Florida: Wingers, 1994. p.984-996.

GODOY, S.N. Psittaciformes (Araras, papagaios, periquito). In: CUBAS, Z.S. et al. Tratado de animais selvagens. Medicina veterinária. São Paulo: Roca, 2007. p.222-251.

GUZMAN, D.S. etal. Evaluating 21-day doxycycline and azithromycin treatments for experimental Chlamydophila psittaci infection in Cockatiels (Nymphicus hollandicus). Journal of Avian Medicine and Surgery, v.24, n.1, p.3545, 2010. Disponível em: <http://www.bioone.org/doi/abs/ 10.1647/2009-009R.1>. Acesso em: 24 mar. 2011. doi: 10.1647/2009-009R.1.

KALETA, E.F.; TADAY, M.A. Avian host range of Chlamydophila spp. based on isolation, antigen detection and serology. Avian Pathology, v.32, n.5, p.435-462, 2003. Disponível em: <http://www.ingentaconnect.com/content/tandf/ cavp/2003/00000032/00000005/art00001>. Acesso em: 24 mar. 2011. doi: 10.1080/03079450310001593613.

LEY, D.H. Performance characteristics of diagnostic tests for avian chlamydiosis. International Journal of Systematic Bacteriology, v.7, p.203-207, 1993. Disponível em: <http:/ /www.jstor.org.proxy-remote.galib.uga.edu/stable/27671089>. Acesso em: 26 mar. 2011. doi: 10.2307/27671089.

LONGBOTTOM, D.; COUlTeR, L.J. Animal Chlamydiosis and zoonotic implications. Journal of Comparative Pathology, v.128, p.217-244, 2003. Disponível em: <http:// www.sciencedirect.com.proxy-remote.galib.uga.edu/ science?_ob=ArticleURL\&_udi=B 6WHW-47 S6 K9 C$1 \& \_$user $=655127 \&$ \&coverDate $=05 \% 2 \mathrm{~F} 31 \% 2 \mathrm{~F} 2003 \&$ \&doc $=1$ \&_fmt=high\&_orig=gateway\&_origin=gateway\&_sort $=\mathrm{d} \&$ _ docanchor $=\&$ view $=c \& \_a c c t=C 000033918 \& \_$version $=1 \& \_u$ rl Version $=0 \&$ u serid $=655127 \&$ md $5=5$ e $01 \mathrm{~d} 28$ 8a39334d96d872c7e51e3c6f9\&searchtype=a>. Acesso em: 26 mar. 2011. doi: 10.1053/jcpa.2002.0629.

MOSCHIONI, C. et al. Pneumonia grave por Chlamydia psittaci. Journal Pneumology, v.27, n.4, p.219-222, 2001. Disponível em: <http://www.scielo.br/scielo.php?pid=S0102$35862001000400008 \&$ script $=$ sci_arttext $>$. Acesso em: 24 mar. 2011. doi: 10.1590/S0102-35862001000400008.

MOULDER, J.W. Interaction of Chlamydia and host cells in vitro. Microbiological Reviews, v.55, p.143-190, 1991. Disponível em: <http://mmbr.asm.org.proxy-remote.galib.uga.edu/ cgi/content/abstract/55/1/143>. Acesso em: 24 mar. 2011.

NASPHV - NATIONAL ASSOCIATION OF STATE PUBLIC HEALTH VETERINARIANS. Compendium of measures to control Chlamydophila psittaci infection among humans (psittacosis) and pet birds (avian chlamydiosis). 2010. Disponível em: <http//avma.org/pubhlth/psittacosis.asp>. Online. Acesso em: 20 jun. 2010.

OLSON, B.J.; TREUTING, W.L. An epidemic of a severe pneumonitis in the Bayou Region of Lousiana. IV. A preliminary note on citology. Public Health Report, v.59, p.1299-1311, 1994. Disponível em: <http://www.jstor.org.proxy-remote.galib.uga.edu/stable/4585048>. Acesso em: 24 mar. 2011. doi: 10.2307/4585048.

PAGE, L.A. Experimental ornithosis in turkeys. Avian Diseases, v.3, p.51-66, 1959. Disponível em: <http:// www.jstor.org.proxy-remote.galib.uga.edu/stable/1587757>. Acesso em: 26 mar. 2011. doi: 10.2307/1587757.

PAGE, L.A.; ERICKSON, K. Serologic evidence of natural and experimental transfers of Chlamydia psittaci between wild and domestic animals. Proceedings Annual Conference Bulletin Wildlife Disease Association, v.5, p.284-290, 1969.

PETROVAY, F.; BALLA, E. Two fatal cases of psittacosis caused by Chlamydophila psittaci. Journal of Medical Microbiology, v.57, p.1296-1298, 2008. Disponível em: <http:// jmm.sgmjournals.org/cgi/content/abstract/57/10/1296>. Acesso em: 24 mar. 2011. doi: 10.1099/jmm.0.2008/001578-0.

RASO, T.F. Detecção de infecção por Chlamydia psitacci em papagaios do gênero Amazona mantidos em cativeiro. 1999. 61f. Dissertação (Mestrado em Medicina Veterinária) Universidade Estadual Júlio de Mesquita Filho, Faculdade de Ciências Agrárias e Veterinárias, Jaboticabal, SP.

RASO, T.F. et al. Evidence of Chlamydophila psittaci infection in captive amazon parrots in Brazil. Journal of Zoo and Wildlife Medicine, v.32, p.118-121, 2002. Disponível em: <http://www.bioone.org/doi/abs/10.1638/10427260(2002)033\%5B0118:EOCPII\%5D2.0.CO\%3B2>. Acesso em: 24 mar. 2011. doi: 10.1638/10427260(2002)033[0118:EOCPII]2.0.CO;2.

RASO, T.F. Chlamydophila psittaci em psitacídeos de vida livre e cativeiro e suas implicações à saúde pública. 2004. 79f. Tese (Doutorado em Medicina Veterinária) - Universidade Estadual Júlio de Mesquita Filho, Faculdade de Ciências Agrárias e Veterinárias, Jaboticabal, SP. 
RASO, T.F.. et al. An outbreak of chlamydiosis in captive bluefronted amazon parrots (Amazona aestiva) in Brazil. Journal of Zoo and Wildlife Medicine, v.35, p.94-96, 2004. Disponível em: http://www.bioone.org.proxyremote.galib.uga.edu/doi/full/10.1638/02-090. Acesso em: 24 mar. 2011. doi: 10.1638/02-090.

RASO, T.F.et al. Chlamydophila psittaci in free-living Blue-fronted Amazon parrots (Amazona aestiva) and Hyacinth macaws (Anodorhynchus hyacinthinus) in the Pantanal of Mato Grosso do Sul, Brazil. Veterinary Microbiology, v.117, p. 235-241, 2006. Disponível em: <http://www.sciencedirect.com.proxyremote.galib.uga.edu/science?_ob=ArticleURL\&_udi=B6TD6$4 \mathrm{KKNJ} 3 \mathrm{~F}-1$ \&_user $=655127 \&$ \&coverDate $=10 \% 2 \mathrm{~F} 31 \% 2 \mathrm{~F} 2006 \&$ _rdoc $=1 \&$ fmt $=$ high \&_orig $=$ gateway \&_origin=gateway $\&$ _ sort $=d \&$ docanchor $=\&$ view $=c \&$ \&_searchStrId $=1694308$ $383 \&$ \&erunOrigin $=$ scholar.google $\&$ _acct $=\mathrm{C} 00003$ 3918 \&_version $=1 \&$ \&urlVersion $=0 \&$ \& userid $=655127$ $\& m d 5=e 68 \mathrm{cf} 24444 \mathrm{c} 6 \mathrm{dbd} 179 \mathrm{f} 3 \mathrm{edd} 041 \mathrm{f} 8 \mathrm{~b} 711 \&$ searchtype $=\mathrm{a}>$. Acesso em: 26 mar. 2011. doi: 10.1016/j.vetmic.2006.06.025.

RASO, T.F. et al. Seroprevalence of antibodies to Chlamydophila psittaci in zoo workers in Brazil. Zoonoses and Public Health, v.57, p.411-416, 2010. Disponível em: <http:// onlinelibrary.wiley.com.proxy-remote.galib.uga.edu/doi/ 10.1111/j.1863-2378.2009.01237.x/full>. Acesso em: 26 mar. 2011. doi: $10.1111 / \mathrm{j} .1863-2378.2009 .01237 . x$.

SACHSE, K. et al. Recent developments in the laboratory diagnosis of chlamydial infections. Veterinary Microbiology, v.135, p.221, 2009. Disponível em: <http://www.sciencedirect.com.proxyremote.galib.uga.edu/science?_ob=ArticleURL\&_udi=B6TD6-
4TFW997-2\&_user $=655127 \&$ _coverDate $=03 \% 2 \mathrm{~F} 16 \% 2 \mathrm{~F} 2009 \&$ _rdoc $=1 \&$ fmt $=$ high\&_orig=gateway\&_origin=gateway\&_so $\mathrm{rt}=\mathrm{d} \&$ _docanchor $=\& \mathrm{view}=\mathrm{c} \&$ _ searchStrId $=1694$ $308982 \&$ \&erunOrigin $=$ scholar.google\&_acct $=$ C $000033918 \&$ \& version $=1 \&$ \& ur 1 Version $=0 \&$ _ u s e r i d $=655127 \&$ m d $5=556 \mathrm{~d} 89965 \mathrm{f}$ 0cbd64447e8e2bb386aa38\&searchtype $=\mathrm{a}>$. Acesso em: 26 mar. 2011. doi: 10.1016/j.vetmic.2008.09.040.

SHEWEN, P.E. Chlamydial infection in animals: a review. Canadian Veterinary Journal, v.21, p.2-11, 1980. Disponível em: <http://www.ncbi.nlm.nih.gov.proxyremote.galib.uga.edu/pmc/articles/PMC $1789659 /$ ?tool=pmcentrez $>$. Acesso em: 26 mar. 2011.

TELFER, B.L. et al. Probable psittacosis outbreak linked to wild birds. Emerging Infectious Diseases, v.1, n.3, p.391397, 2005. Disponível em: http://journal.shouxi.net/qikan/ article.php?id=223293. Acesso em: 26 mar. 2011.

VANROMPAY, D. et al. Chlamydia pisittaci infections: a review with emphasis on avian chlamydiosis. Veterinary Microbiology, v.45, p.93-119, 1995. Disponível em: <http:/ /www.sciencedirect.com.proxy-remote.galib.uga.edu/ science?_ob=ArticleURL\&_udi=B6TD6-3YS91 W0D\&_user $=655127 \&$ \& coverDate $=07 \% 2$ F $31 \% 2 \mathrm{~F} 1$ $995 \& \_r d o c=1 \& \_f m t=h i g h \&$ orig=gateway \&_origin=gateway \& _sort $=d \&$ docanchor $=\& v i e w=c \&$ searchStrId $=16943199$ 67\&_rerunOrigin $=$ scholar.google $\& \_a c c t=C 000033918 \& \_v e r s i o n=1 \&$ _urlVersion $=0 \&$ _ us e rid $=655127 \&$ m d $5=$ f 7 $6 \mathrm{c} 6 \mathrm{cac} 94 \mathrm{~d} 98 \mathrm{f} 489338 \mathrm{f} 8287 \mathrm{aa} 506 \mathrm{f} 6 \&$ searchtype $=\mathrm{a}>$. Acesso em: 26 mar. 2011. doi: 10.1016/0378-1135(95)00033-7. 